\title{
Experiment, theory, and simulation of the evacuation of a room without visibility
}

\author{
Motonari Isobe, ${ }^{1}$ Dirk Helbing, ${ }^{2}$ and Takashi Nagatani ${ }^{1}$ \\ ${ }^{1}$ Department of Mechanical Engineering, Division of Thermal Science, Shizuoka University, Hamamatsu 432-8561, Japan \\ ${ }^{2}$ Institute for Economics and Traffic, Dresden University of Technology, D-01062 Dresden, Germany
}

(Received 5 June 2003; revised manuscript received 27 January 2004; published 17 June 2004)

\begin{abstract}
We study the evacuation process from a smoky room by means of experiments and simulations. People in a dark or smoky room are mimicked by "blind" students wearing eye masks. The evacuation of the disoriented students from the room is observed by video cameras, and the escape time of each student is measured. We find that the disoriented students exhibit a distinctly different behavior compared to a situation in which people can see their environment. Our experimental results are related to a theoretical approach and reproduced by an extended lattice gas model taking into account the empirically observed behavior. Our particular focus is on the mean value and distribution of escape times. For a large number of people in the room, the escape time distribution is wide because of jamming. Surprisingly, adding more exits does not improve the situation in the expected way, since most people use the exit that is discovered first, which may be viewed as a kind of herding effect based on nonlocal, but direct acoustic interactions. Moreover, the average escape time becomes minimal for a certain finite number of people in the dark or smoky room. These nonlinear effects have practical implications for emergency evacuation and the planning of safer buildings.
\end{abstract}

DOI: 10.1103/PhysRevE.69.066132

PACS number(s): 89.40.-a, 05.70.Fh, 05.90.+m

\section{INTRODUCTION}

During the last decade, many observed self-organization phenomena in traffic flows [1-5] and pedestrian streams [6-10] have been successfully reproduced with physical methods. This has not only stimulated research in granular, biological, and colloid physics [11-13]. It has also encouraged physicists to study evacuation processes [14-16], since it has been shown that many aspects of crowd stampedes can be understood by driven many-particle models $[17,18]$. The empirical observations have many common features with driven granular media.

Evacuation processes have been studied by the use of various simulation models. The typical models of pedestrian motion are based on molecular dynamics methods $[6,17,18]$, lattice gas models [7,19-25], or cellular automata $[9,15,16,26,27]$. In simulations of evacuation processes, it has been mostly assumed that visibility is good. However, this is often not the case during fire emergencies, as smoke or a failure of the electrical power supply reduce the orientation significantly. Because of the toxic effects of smoke, fast evacuation is particularly important, but little is known about the behavior of people under conditions of bad or no visibility.

In this study, we will therefore focus on the investigation of the evacuation process from a room without visibility. In favor of video recordings, we mimic this situation by requiring our test persons to wear eye masks. This allows us to study not only qualitative features, but also quantitative outcomes, which is relevant for reliable predictions of evacuation times and the planning of safer buildings or pedestrian facilities.

From our video recordings, we have evaluated the characteristic behavior and the escape times in well-controlled experiments (see Sec. II). Section III explains some of the findings by means of a theoretical approach, while Sec. IV presents an extended lattice gas model of pedestrian flows with model parameters calibrated to the data. Despite the simplicity of the model, it can successfully reproduce the characteristic escape behavior under conditions of no visibility and the empirical escape times in a semiquantitative way. Moreover, we identify two interesting effects: First, the average escape time becomes minimal for a specific finite number of people, who are initially in the room. Second, adding more exits does not increase the efficiency of evacuation in the expected way.

\section{EXPERIMENT}

We have experimentally studied the evacuation of blind students from an empty classroom, which is schematically illustrated in Fig. 1. Each student wore an eye mask. The exact width of the classroom was $W=4.2 \mathrm{~m}$ and its length $L=5.5 \mathrm{~m}$. There were no obstacles in the classroom; i.e., desks and chairs were moved aside (to form the boundary). Moreover, the room had one exit of width $0.5 \mathrm{~m}$. Two video cameras 1 and 2 were located within and in front of the classroom. One of the cameramen was able to observe all the students by video camera 1 . The other cameraman could observe the students who escaped through the exit by video camera 2. We have investigated two cases:

(a) the escape of only one disoriented student with an eye mask,

(b) the evacuation of ten-disoriented students.

Correspondingly, at time $t=0$, there were either (a) one student or (b) ten students in the classroom, and each student was standing at a random place within a central area of the room. Before the experiment, all students were forced by the cameraman to turn around themselves. In the result, they lost their directional orientation. All students moved to seek for the exit as soon as cameraman 1 shouted a word of command. The evacuation process was then recorded by the two video cameras. Cameraman 1 changed his position in every 


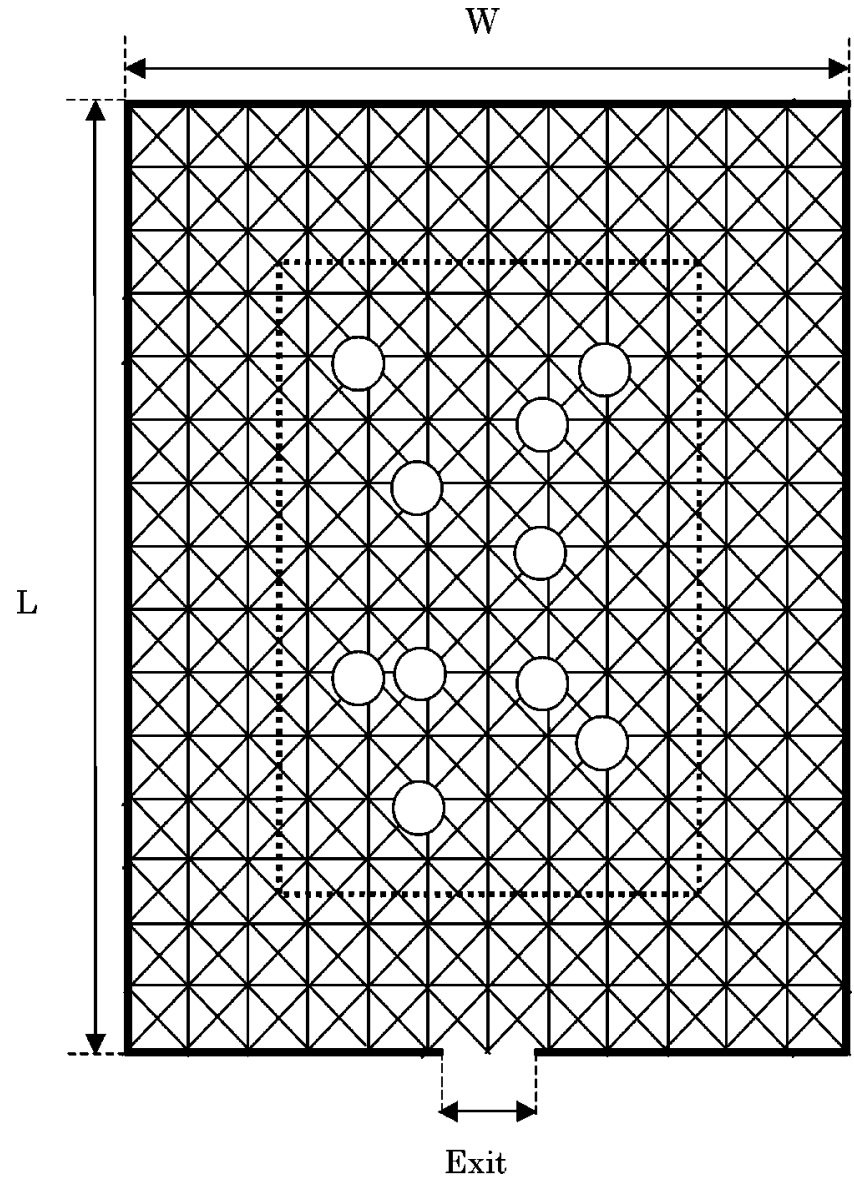

FIG. 1. Schematic illustration of the classroom of width $W$ $=4.2 \mathrm{~m}$ and length $L=5.5 \mathrm{~m}$, in which desks, chairs, and other obstacles have been moved aside. There is only one exit in the front of the classroom, the width of which is $0.5 \mathrm{~m}$. Two video cameras 1 and 2 were located within and in front of the classroom to record the motion of all test persons. In our experiments with ten persons, everyone started in the central area indicated by the dashed line. White circles represent the initial (off-lattice) positions of the test persons, while the simulation model approximates their motion with a lattice gas model in which particles hop between the discrete sites of a square-diagonal lattice with $W=11$ sites in the $x$ direction and $L=14$ sites in the $y$ direction (see also Figs. 6, 9, and 10). The possible directions of motion are indicated by the solid lines (see Fig. 6), while the elementary cells are $W \times L$ squares. When the site next to the exit is reached, the corresponding walker is removed immediately in analogy to our experimental observations.

experiment in order not to indicate the position of the exit. Figures 2 and 4 show that the test persons could actually not guess the direction of the exit.

We have first studied the case (a) of single disoriented students with eye masks. By careful analysis of the video recordings, we have determined the trajectory and escape time of each student. The individual escape time was defined as the time elapsed between the shouting of the command and the moment when the respective student left the room through the exit. Figure 2 shows four typical trajectories of a single student until he successfully escaped from the room. His face direction at the initial position is indicated by an arrow. At first, the student turned slightly, and then he moved

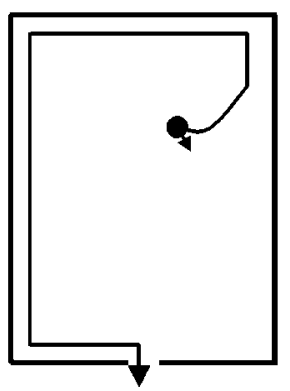

$t_{e}=39 \mathrm{sec}$

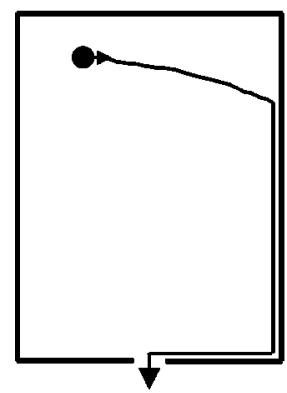

$t_{e}=23 \mathrm{sec}$

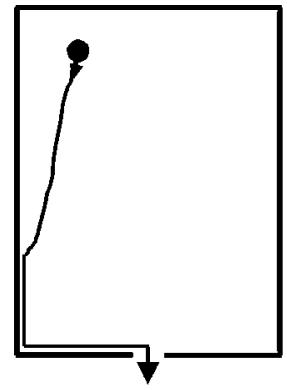

$t_{e}=25 \mathrm{sec}$

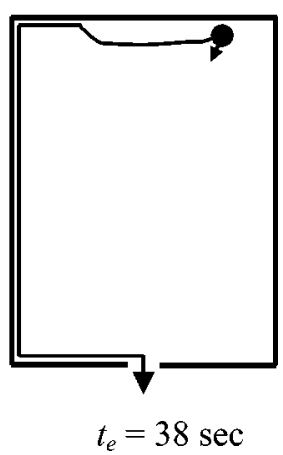

FIG. 2. Four typical trajectories of students until they successfully escape from the room. Their face direction at the initial position is indicated by an arrow. Below each trajectory, the escape time obtained from the experiment is indicated.

slowly towards one of the walls of the room. When he touched the boundary, he followed it, after he had chosen the right-hand or left-hand direction at random, because of the loss of directional memory. As soon as he found the exit, he left the room. Note that the behavior of following the wall differs significantly from the behavior assumed in the evacuation simulations of Ref. [17], where individuals "hitting" a wall where "reflected." It is, therefore, interesting and important to check, whether the conclusions for the evacuation of a group of people are to be revised. It will turn out that our simulations are an independent, experimentally supported confirmation of the previous findings, which were obtained for a hypothetical pedestrian behavior under conditions of smoke.

In Fig. 2, the escape time obtained from the experiment is shown below four representative trajectories. One can see that the disoriented students sometimes take the shorter way and at other times the longer way. Thus, the escape time depends highly on the randomly chosen direction. We have repeated the experiment 10 times with ten different students. The mean escape time of $t_{e}=31.5 \mathrm{sec}$ was obtained by averaging over all experiments, but the variance was large, as expected.

Next, we have studied the case (b) of ten disoriented students with eye masks. Initially, the ten students were standing at random places close to the center of the room. By careful analysis of our video recordings, we have determined the trajectories and escape times of all ten students. Figure 3 shows a photo of the evacuation of students from the room at 


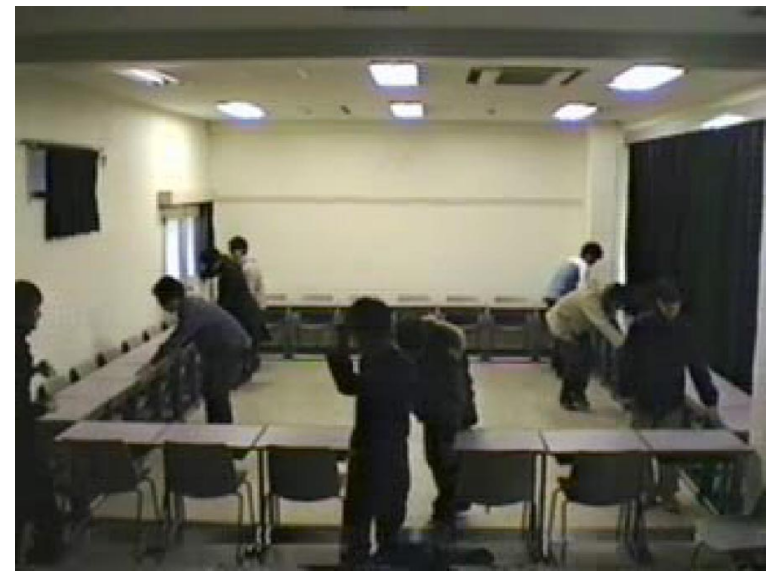

FIG. 3. Photo of the evacuation of students from the room at $t$ $=16 \mathrm{sec}$.

time $t=16 \mathrm{sec}$. The students move along the boundary. Figure 4 shows the time evolution of the evacuation process for ten students. The patterns (a)-(d) were obtained at times $t$ $=0 \mathrm{sec}, 5 \mathrm{sec}, 10 \mathrm{sec}$, and $15 \mathrm{sec}$. Numbered circles represent the ten disoriented students, whose face directions are indicated by arrows. The students turned slightly at first and moved slowly towards one of the walls of the room. When they touched a wall, they followed it in one of the two pos-

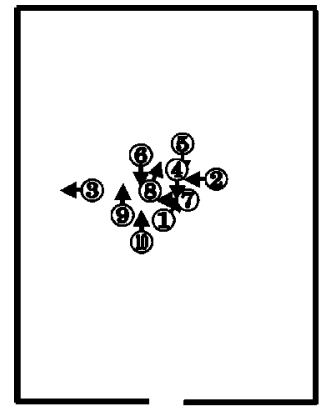

(a)

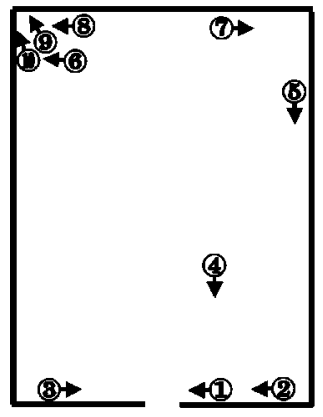

(c)

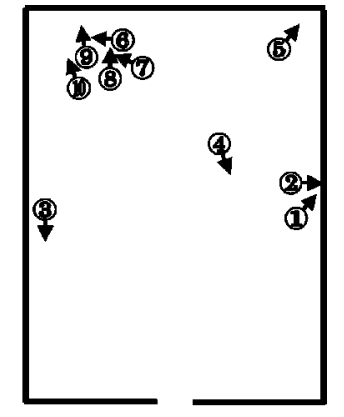

(b)

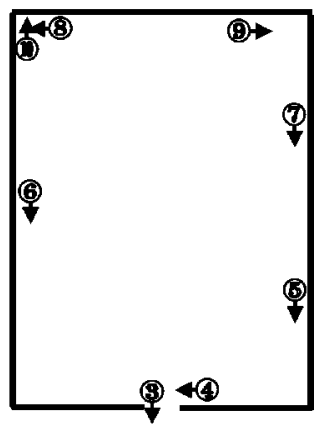

(d)
FIG. 4. Time evolution of the evacuation process of ten students without visibility of the exit. The patterns (a)-(d) were obtained at $t=0 \mathrm{sec}, 5 \mathrm{sec}, 10 \mathrm{sec}$, and $15 \mathrm{sec}$. Numbered circles represent ten disoriented students with eye masks, whose face directions are indicated by arrows.

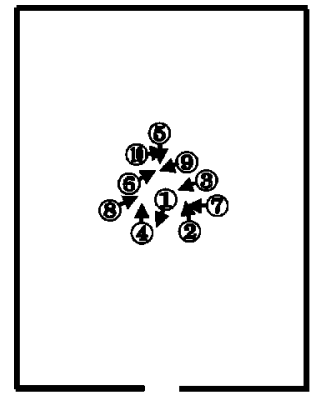

(a)

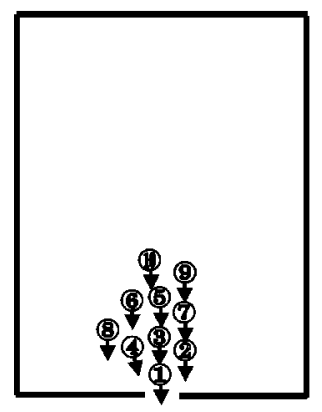

(c)

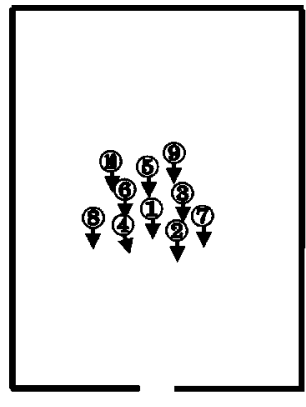

(b)

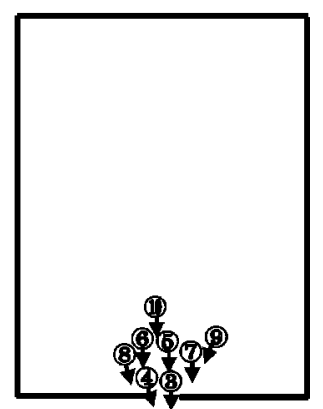

(d)
FIG. 5. Time evolution of the evacuation process for ten students in a room with normal visibility. The patterns (a)-(d) were obtained at $t=0 \mathrm{sec}, 1 \mathrm{sec}, 3 \mathrm{sec}$, and $5 \mathrm{sec}$. Their face directions are indicated by arrows.

sible directions. However, as soon as one or two students managed to leave the room, the remaining students located the exit acoustically. This was possible, because moving in a room always produces some noise, so that leaving the room is connected with the disappearance of a noise source. (In emergency situations, people would even tell each other when they have found the way out.) Therefore, the remaining students inverted their direction, if appropriate, while continuing to follow the boundary. When a student met another one, they went together along the wall into the same direction. They left the room as soon as they reached the exit.

We have determined the escape times of all students by careful analysis of our video recordings of ten repetitions of the experiment. The average escape time of the ten students was $22.1 \mathrm{sec}$. However, the escape time of the fastest student was $9.8 \mathrm{sec}$, while the value for the slowest student was $34.3 \mathrm{sec}$. Thus, the distribution of escape times was again rather wide. Notice that the average escape time of $22.1 \mathrm{sec}$ for ten students is significantly lower than the $31.5 \mathrm{sec}$ for a single student. This reduced escape time is due to the fact that, when one or two students managed to leave the room successfully, the remaining students noticed the location of the exit and inverted their direction, if appropriate.

For comparison, we performed an escape experiment for ten students without eye masks corresponding to a room with normal visibility. Figure 5 shows the time evolution of the evacuation process of all ten students. The patterns (a)-(d) were obtained at times $t=0 \mathrm{sec}, 1 \mathrm{sec}, 3 \mathrm{sec}$, and $5 \mathrm{sec}$. Face 


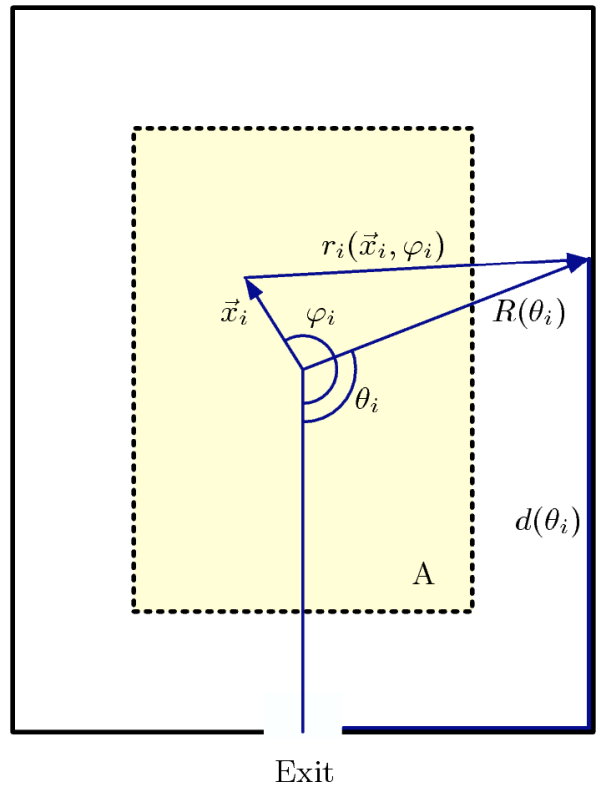

FIG. 6. Illustration of the variables occuring in the theoretical approach to our evacuation experiment from a smoky room (see text for details).

directions are indicated by arrows. The students turned instantly towards the exit and moved into its direction very fast. When the arrival rate of students exceeded the capacity of the exit, students were queueing; i.e., a crowd of students was forming in front of the exit due to jamming. The average escape time of the ten students was $6.54 \mathrm{sec}$. This value is significantly lower than the $22.1 \mathrm{sec}$ for ten disoriented students with eye masks.

Therefore, the evacuation process from a room with no visibility is qualitatively and quantitatively different from the evacuation under normal conditions. When there is sufficient orientation-e.g., a little bit of light indicating the direction of the exit-people will head directly towards the exit based on their visual perception as in the experiment shown in 5. If smoke is dense or there is no light because of a blackout of electricity, the situation corresponds to the experiment represented by Fig. 4, where people use acoustic and tactile perceptions instead.

\section{THEORETICAL APPROACH}

For a theoretical description, we will define the geometrical center of gravity of the room as the origin of the coordinate system. To parametrize the boundary, we will use polar coordinates. $R(\theta)$ shall denote the distance of the wall from the origin as a function of the polar angle $\theta$, where 0 $\leqslant \theta<2 \pi$. The center of the exit shall be located at $\theta=0$. Now, let $\vec{x}_{i}=\left(x_{i 1}, x_{i 2}\right)^{\text {tr }}$ be the initial position of person $i$ and $\vec{e}_{i}=\left(\sin \varphi_{i}, \cos \varphi_{i}\right)^{\text {tr }}$ the initial direction of motion (see Fig. $6)$.

For the sake of simplicity, we will assume that the individual moves with a constant velocity $v_{i}$ into direction $\vec{e}_{i}$ until it hits the boundary for the first time. This will happen after a distance $r_{i}=r_{i}\left(\vec{x}_{i}, \varphi_{i}\right)$, which is determined by the vectorial relationship

$$
R\left(\theta_{i}\right)\left(\begin{array}{c}
\sin \theta_{i} \\
\cos \theta_{i}
\end{array}\right)=\left(\begin{array}{c}
x_{i 1} \\
x_{i 2}
\end{array}\right)+r_{i}\left(\begin{array}{c}
\sin \varphi_{i} \\
\cos \varphi_{i}
\end{array}\right)
$$

Herein, the function $R(\theta)$ is given, and $\vec{x}_{i}, \varphi_{i}$ are determined by the initial condition (see Fig. 6). Therefore, the set of two equations is sufficient to calculate $\theta_{i}$ and $r_{i}$. These quantities determine the individual escape time $t_{\mathrm{e}, i}=t_{i}^{1}+t_{i}^{2}$ in the following way: The first contribution is given by the distance $r_{i}$ divided by the velocity of motion $v_{i}$, which implies $t_{i}^{1}$ $=r_{i} / v_{i}$. The second contribution is given by the distance $d\left(\theta_{i}\right)$ to the exit when following the wall in the direction of decreasing angles $\theta_{i}$ (see Fig. 6): If the walking speed along the wall is $w_{i}$, we find an additional escape time of $d\left(\theta_{i}\right) / w_{i}$ in the direction of decreasing angles, but $\left[d(2 \pi)-d\left(\theta_{i}\right)\right] / w_{i}$ into the opposite direction, which is randomly chosen with probability $1 / 2$. Altogether we have the relation

$$
\begin{aligned}
t_{\mathrm{e}, i}\left(\vec{x}_{i}, \varphi_{i}\right) & =\frac{r_{i}\left(\vec{x}_{i}, \varphi_{i}\right)}{v_{i}}+\frac{1}{2}\left[\frac{d\left(\theta_{i}\right)}{w_{i}}+\frac{d(2 \pi)-d\left(\theta_{i}\right)}{w_{i}}\right] \\
& =\frac{r_{i}\left(\vec{x}_{i}, \varphi_{i}\right)}{v_{i}}+\frac{d(2 \pi)}{2 w_{i}},
\end{aligned}
$$

where $d(2 \pi)$ corresponds to the perimenter of the room. If all individuals $i$ would start from the center $\vec{x}_{i}=\overrightarrow{0}$ of the room and the boundary of the room would be a circle of radius $R$, the result would simply be $t_{\mathrm{e}, i}=R\left[1 / v_{i}+\pi / w_{i}\right]$.

When we want to determine the average escape time $\overline{t_{e}}$, we need to average over all individuals $i$, in particular over all possible initial conditions $\vec{x}_{i}$ and $\varphi_{i}$. If the area covered by all admissible starting points $\vec{x}_{i}$ is denoted by $A$, we find

$$
\begin{aligned}
\overline{t_{e}}= & \frac{1}{A} \int_{A} d^{2} x_{i} \frac{1}{2 \pi} \int_{0}^{2 \pi} d \varphi_{i} \int_{0}^{\infty} d v_{i} p_{v}\left(v_{i}\right) \int_{0}^{\infty} d w_{i} p_{w}\left(w_{i}\right) \\
& \times\left[\frac{r_{i}\left(\vec{x}_{i}, \varphi_{i}\right)}{v_{i}}+\frac{d(2 \pi)}{2 w_{i}}\right] .
\end{aligned}
$$

Here, $p_{v}\left(v_{i}\right)$ denotes the probability density of the occurrence of velocity $v_{i}$, and $p_{w}\left(w_{i}\right)$ the corresponding probability density for $w_{i}$.

We can immediately see that formula (3) can only be solved numerically (apart from a few special cases). Therefore, it is of limited use. Things become even more complicated if the motion is modeled as a biased random walk, which requires one to solve a first-passage time problem. We can, however, see that the result generally depends on the shape of the room via the dependence on $r_{i}$. This can account for the nonsmooth escape time distribution of a single person found in Sec. IV. Moreover, the escape time depends on the circumference $d(2 \pi)$, which is particularly interesting for more or less fractal boundaries. Also note that the existence of a second exit at $\theta_{i}=\pi$ would modify the formula according to 


$$
\begin{aligned}
\overline{t_{e}}= & \frac{1}{A} \int_{A} d^{2} x_{i} \frac{1}{2 \pi} \int_{0}^{2 \pi} d \varphi_{i} \int_{0}^{\infty} d v_{i} p_{v}\left(v_{i}\right) \int_{0}^{\infty} d w_{i} p_{w}\left(w_{i}\right) \\
& \times\left[\frac{r_{i}\left(\vec{x}_{i}, \varphi_{i}\right)}{v_{i}}+\frac{d(\pi)}{2 w_{i}}\right],
\end{aligned}
$$

where $d(2 \pi)$ was replaced by $d(\pi)$. Therefore, the average escape time of a single person is reduced by the availability of a second door, as expected. However, if the two exits would be located just next to each other, the average escape time would stay almost unchanged compared to Eq. (3).

Since we cannot solve the above formulas analytically and as the possibilities for experiments are limited for practical reasons, we will now formulate a lattice gas model. It will allow us to understand the implications of our experimental observations in more detail and to assess the efficiency of escape and the safety of buildings under different conditions, in particular under conditions of no visibility.

\section{MANY-PARTICLE SIMULATION}

In the following, we will describe a simple model which allows to reproduce our experimental findings for the evacuation of a dark or smoky room in a semiquantitative way. We will simulate the pedestrian flow by the use of a lattice gas model, but it would be also possible to apply the social force model of pedestrian behavior $[6,17,18]$. We have implemented the following characteristics of disoriented people:

(1) Each walker turns slightly at first and moves towards one of the walls.

(2) He chooses the right-hand or left-hand direction at random as soon as he reaches a wall.

(3) Afterwards, he moves along the wall.

(4) When one walker has managed to leave the room, the remaining walkers turn into the direction of the exit as well, while continuing to follow the wall.

Each disoriented student is represented by a walker on a square-diagonal lattice with $L \times W$ sites reflecting the classroom. We choose the lattice spacing as $0.4 \mathrm{~m}$, since the typical space occupied by a pedestrian in a dense crowd is about $0.4 \mathrm{~m} \times 0.4 \mathrm{~m}$. Correspondingly, we use $L=14$ and $W=11$. The classroom is connected to the outer space through a single exit represented by one site.

Figure 1 shows a schematic illustration. An open circle represents a student in the classroom. In reasonable agreement with the empirical observations, we assume that each walker performs a biased-random walk on the squarediagonal lattice until he reaches the boundaries of the room [28]. Initially, each walker chooses randomly one of the eight directions on the square-diagonal lattice. The walker is then biased with respect to this direction, which represents the desired walking direction of the student. The biased random walker is allowed to move not only to the nearest-neighbor sites, but also to the next-nearest-neighbor sites in the diagonal directions.

Figure 7 illustrates two of all possible configurations of a biased random walker on the square-diagonal lattice, where

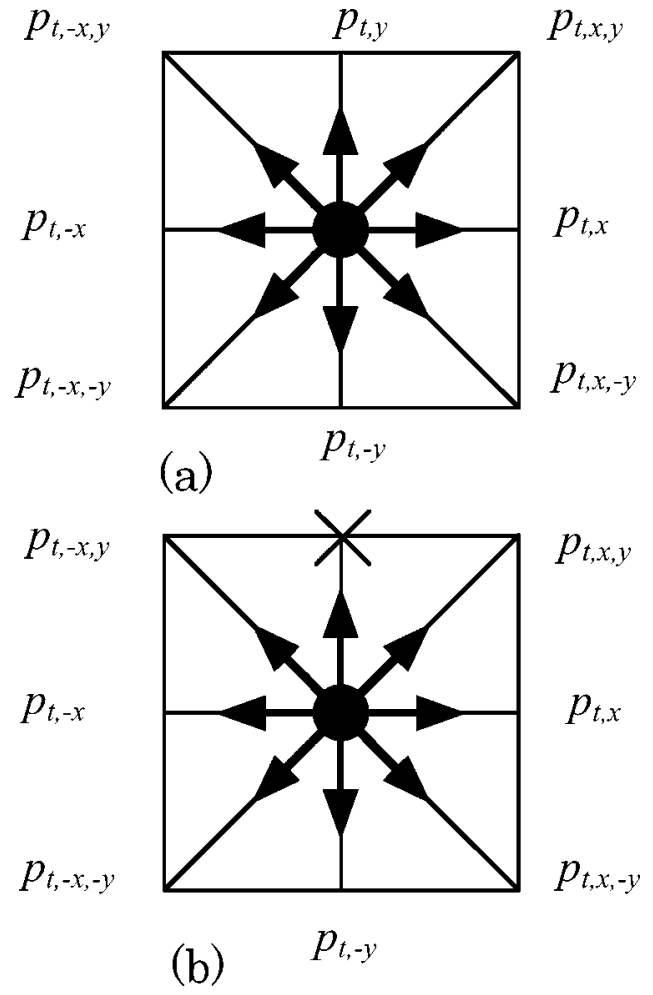

FIG. 7. Two of all possible configurations of a biased-random walker on the square-diagonal lattice, where bias is applied to the upward direction. Configuration (a) shows the situation of being able to move to all the nearest-neighbor and next-nearest-neighbor sites. The arrows indicate the possible directions in which the walker can move. Configuration (b) shows the situation in which another walker occupies one of the nearest-neighbor sites, which is indicated by a cross.

bias is assumed into the upward direction. Configuration (a) shows the situation of being able to move to all the nearestneighbor and next-nearest-neighbor sites, when these are not occupied by other walkers. The arrows indicate the possible directions in which the walker can move. The transition probabilities of the walker into the eight directions are given by

$$
\begin{gathered}
p_{t, y}=D / 3+(1-D) / 8=p_{t, y}^{(\mathrm{a})}, \\
p_{t, x, y}=p_{t,-x, y}=D / 6+(1-D) / 8=p_{t, x, y}^{(\mathrm{a})}, \\
p_{t, x}=p_{t,-x}=D / 9+(1-D) / 8=p_{t, x}^{(\mathrm{a})}, \\
p_{t,-y}=p_{t, x,-y}=p_{t,-x,-y}=D / 27+(1-D) / 8=p_{t,-y}^{(\mathrm{a})},
\end{gathered}
$$

where $D$ is the parameter representing the bias, $p_{t, y}$ is the transition probability in the $y$ direction, and the superscript (a) refers to configuration (a). Specifically, $p_{t, y}^{(a)}$ indicates the transition probability into the $y$ direction in configuration (a), $p_{t, x, y}^{(\mathrm{a})}$ the transition probability into the diagonal $x-y$ direction, etc. The sum of the transition probabilities equals 1 , because the probability not to move was assumed to be zero. In the following simulation, we set $D=0.99$. The sum of the tran- 
sition probabilities with which a particle hops forward or laterally is 0.89 , where $p_{t, y}^{(\mathrm{a})}=0.33, p_{t, x, y}^{(\mathrm{a})}=0.17$, and $p_{t, x}^{(\mathrm{a})}$ $=0.11$. Since one cell (lattice spacing) corresponds to $0.4 \mathrm{~m}$ and unit time to $1 \mathrm{sec}$, the average velocity of moving forward or sidewards is $0.35 \mathrm{~m} / \mathrm{sec}$. This value agrees quite well with the average speed $0.33 \mathrm{~m} / \mathrm{sec}$, obtained from the experiment. Therefore, we identify one time step in our simulations with one second.

Figure 7(b) shows the configuration in which another walker occupies one of the nearest-neighbor sites. The location of the other walker is indicated by a cross. Then, the transition probabilities into the remaining seven directions are given by

$$
\begin{gathered}
p_{t, x, y}=p_{t,-x, y}=p_{t, x, y}^{(\mathrm{a})}\left(1+\frac{p_{t, y}^{(\mathrm{a})}}{1-p_{t, y}^{(\mathrm{a})}}\right), \\
p_{t, x}=p_{t,-x}=p_{t, x}^{(\mathrm{a})}\left(1+\frac{p_{t, y}^{(\mathrm{a})}}{1-p_{t, y}^{(\mathrm{a})}}\right), \\
p_{t,-y}=p_{t, x,-y}=p_{t,-x,-y}=p_{t,-y}^{(\mathrm{a})}\left(1+\frac{p_{t, y}^{(\mathrm{a})}}{1-p_{t, y}^{(\mathrm{a})}}\right) .
\end{gathered}
$$

Again, the sum of the transition probabilities equals 1, because the probability not to move was set to 0 . For the other possible configurations, the transition probabilities of walkers are specified analogously. Therefore, the explicit expressions are omitted to safe manuscript space.

In our simulations, we assume that initially (at time $t=0$ ), all disoriented students stand at some location in the classroom without any directional memory. In the next time step $(t=1)$, each student starts moving in order to escape from the room. Until he reaches the boundary (a wall), he performs a biased-random walk according to the model sketched above. For each random walker, we assume a constant bias into the desired direction defined by the first step. All walkers are updated once every time step in a random sequential way as, in reality, the students move asynchronously. More specifically, we use a shuffled update, as it avoids dealing with situations where two particles want to move to the same site in one time step. As the students did not see each other, we do not model reaction times.

When a walker reaches the boundary, he chooses the direction to the left or to the right randomly with probability $1 / 2$. After this choice, he moves along the wall. Furthermore, when one walker manages to leave the room, the remaining walkers adopt their desired direction to the direction of the exit. Excluded volume effects are taken into account by preventing multiple occupation of the same site; i.e., each site contains only one individual walker or it is empty.

When a walker reaches the site next to the exit, he is immediately removed from the simulation. This corresponds to the observed behavior in the experiment, where students moved instantly out of the room when they reached the exit. As no clogging effect was observed in the experiments described here, a more detailed model of the dynamics at the exit was not necessary. (For different kinds of experiments see Ref. [10].)

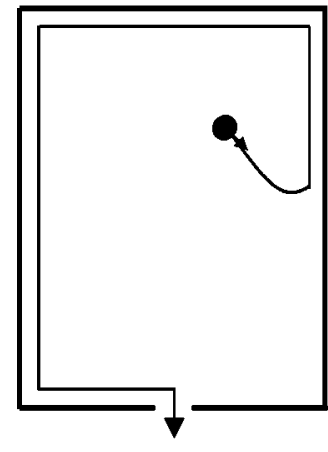

$t_{e}=41 \mathrm{sec}$

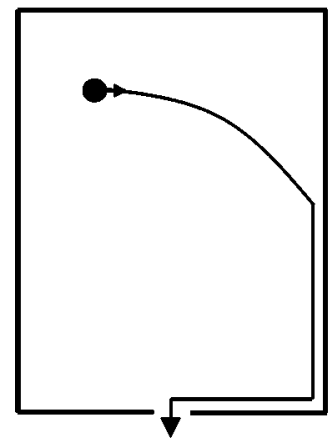

$t_{e}=24 \mathrm{sec}$

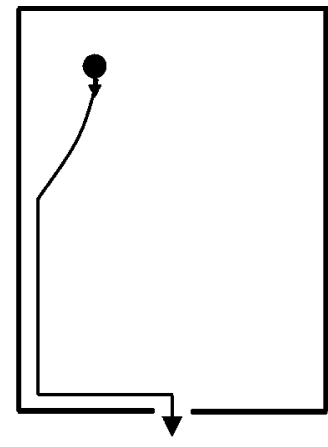

$t_{e}=23 \mathrm{sec}$

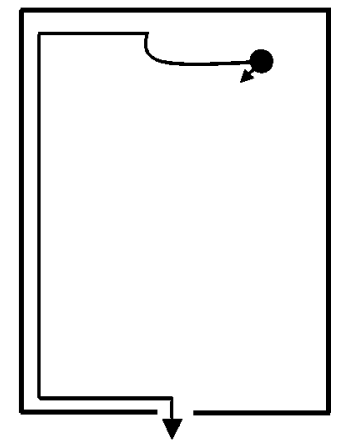

$t_{e}=32 \mathrm{sec}$
FIG. 8. Representative trajectories obtained from the simulation when there is only one walker. The escape times obtained are shown below the trajectories.

Our numerical results are displayed in Figs. 8-14. Figure 8 shows some representative trajectories when there is only one walker. The related escape times for trajectories (a)-(d) were (a) $t_{e}=41 \mathrm{sec}$, (b) $t_{e}=23 \mathrm{sec}$, (c) $t_{e}=24 \mathrm{sec}$, and (d) $t_{e}$ $=32 \mathrm{sec}$. These values are indicated below the trajectories. Like the experimentally obtained trajectories displayed in Fig. 2, the simulated ones show random variations around a dominating direction, until the walker hits the wall and moves straight along the boundary. Moreover, the escape times of simulated trajectories are comparable to those of similarly looking empirical trajectories. Obviously, the es-

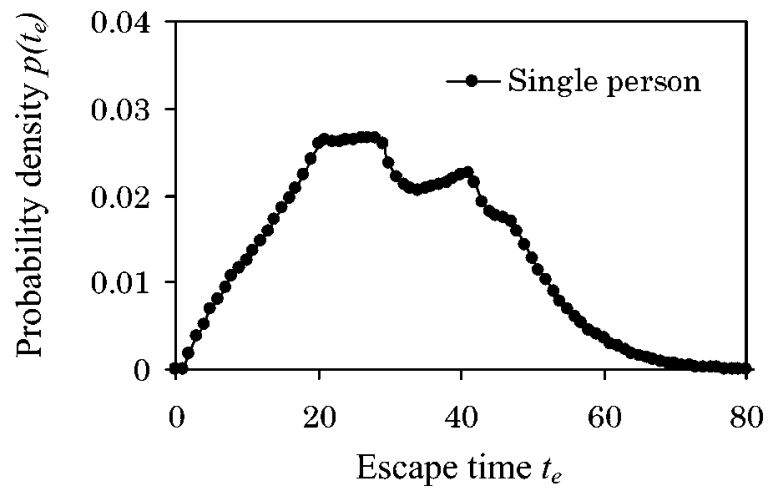

FIG. 9. Plot of the probability density of the simulated escape time for a single walker. 


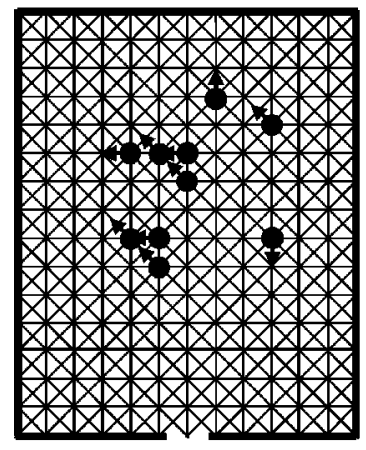

(a)

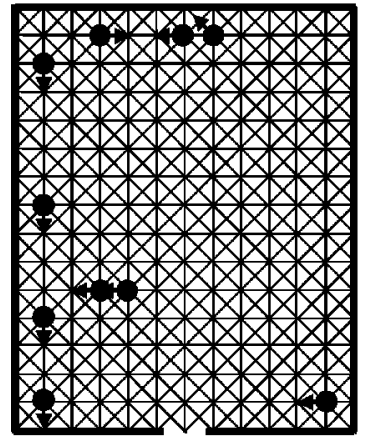

(c)

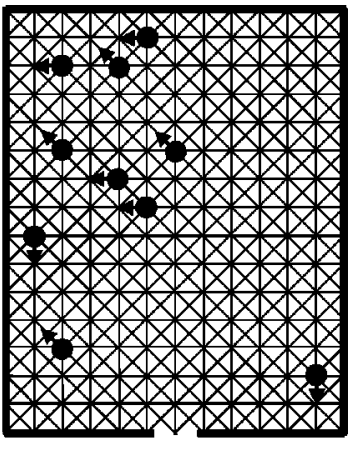

(b)

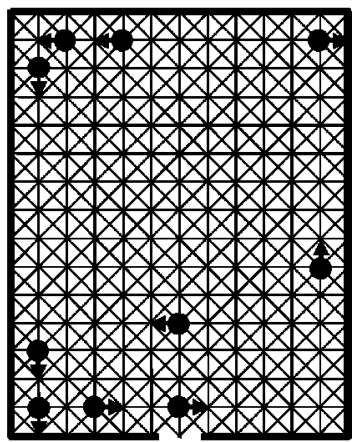

(d)

FIG. 10. Simulated time evolution of the evacuation process of ten persons. The patterns (a)-(d) were obtained at $t=0 \mathrm{sec}, 5 \mathrm{sec}$, $10 \mathrm{sec}$, and $15 \mathrm{sec}$. Solid circles represent ten students, whose face directions are indicated by arrows.

cape time depends very much on the initial position and direction of the person, which is consistent with the analytical result (2). The mean value of $30.1 \mathrm{sec}$ for the simulated escape time was obtained by averaging over 100000 samples and compares quite well with the average escape time of $31.5 \mathrm{sec}$ in our experiments.

Figure 9 shows the probability density of escape times for one student, obtained from 100000 simulation runs. The probability density exhibits a rather wide distribution, but the mean escape time agrees well with the one obtained from the experiment. Note that the nonsmooth distribution of the escape time for a single person is not due to an insufficient number of simulation runs, but due the rectangular shape of the room (see Sec. III). This shape effect is neutralized by interactions among individuals, as is reflected by the smooth escape time distributions for a finite number of persons.

Figure 10 shows the time evolution of the evacuation process of ten persons according to our simulations. The representative patterns (a)-(d) were obtained at times $t=0 \mathrm{sec}$, $5 \mathrm{sec}, 10 \mathrm{sec}$, and $15 \mathrm{sec}$. Solid circles represent ten walkers, whose face directions are indicated by arrows. The behavior of the ten simulated walkers was qualitatively the same as the one observed in our experiments (see Fig. 4). The presence of other persons reduced the mean value of the escape time as in our experiments, but with $27.1 \mathrm{sec}$, the simulation result was a pessimistic estimate of the experimental value.

Similarly, Fig. 11 shows the simulated time evolution of the evacuation process for 20 persons. The patterns (a)-(d)

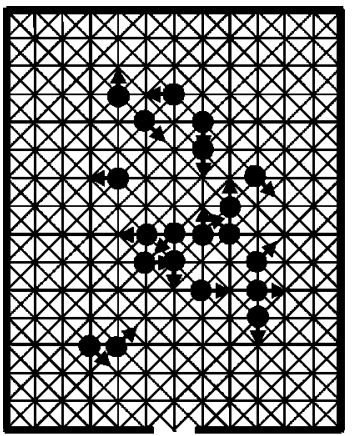

(a)

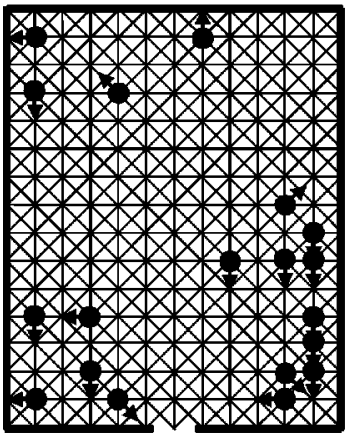

(c)

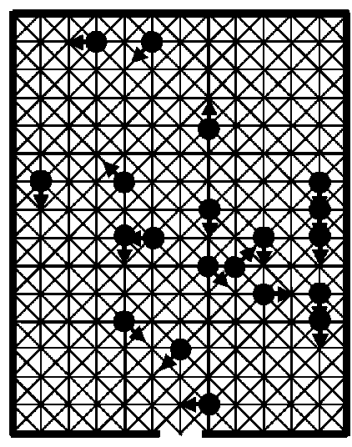

(b)

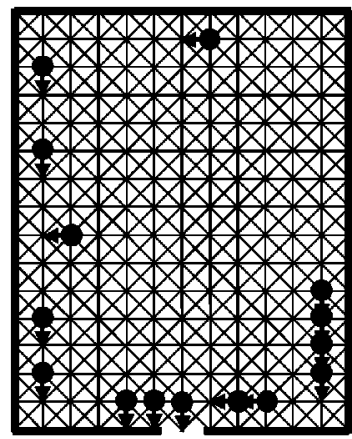

(d)
FIG. 11. Simulated time evolution of the evacuation of 20 persons. The patterns (a)-(d) were obtained at $t=0 \mathrm{sec}, 5 \mathrm{sec}, 10 \mathrm{sec}$, and $20 \mathrm{sec}$.

were obtained at times $t=0 \mathrm{sec}, 5 \mathrm{sec}, 10 \mathrm{sec}$, and $20 \mathrm{sec}$. The behaviors of the 20 simulated walkers was similar to those of 10 persons. However, jamming appeared near the exit at $t=20 \mathrm{sec}$, because the arrival rate of walkers exceeded the capacity of the exit.

We have determined the probability density distribution of escape times of a finite number of walkers from 100000 simulation runs. Figures 12(a)-12(d) show, respectively, the plots of the probability density of escape times for 5, 10, 15, and 20 persons. In each figure, the probability density distributions are shown for the 1st, 5th, 10th, 15th, and 20th persons. By visual inspection of the escape time distributions in Fig. 12(d) for 20 persons, one would say that the distribution of first person is not Gaussian, but the distributions of 5th, 10th, and 15th persons look similar to Gaussian distributions. With an increasing number of walkers, the probability density distribution of escape times for the $n$th person becomes narrower: i.e., the variance decreases. The reason for the different shape of the distribution for different group sizes is the different average escape time of the first leaving person.

Figure 13 shows the probability density distributions of the overall escape times for all persons in the cases of 1, 5, 10,15 , and 20 walkers. Up to 10 walkers, the probability density distribution becomes slightly narrower with an increasing number of walkers, but the distribution becomes rather wide when the number of walkers exceeds 10. For fewer than 10 walkers, the efficiency of the escape is enhanced by the presence of other persons who may discover 


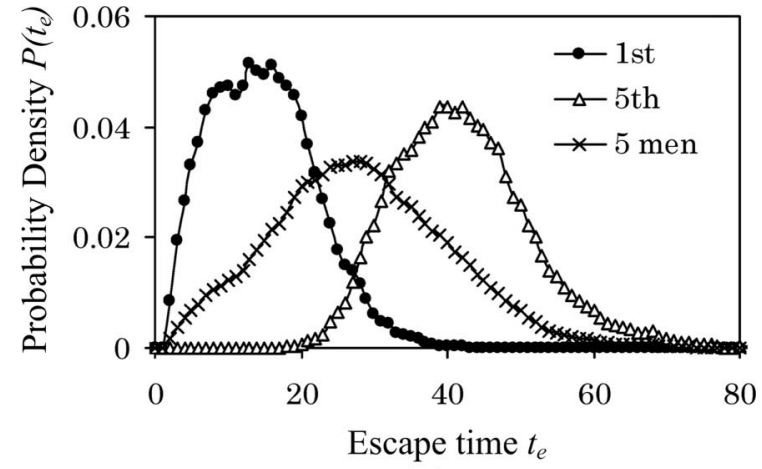

(a)

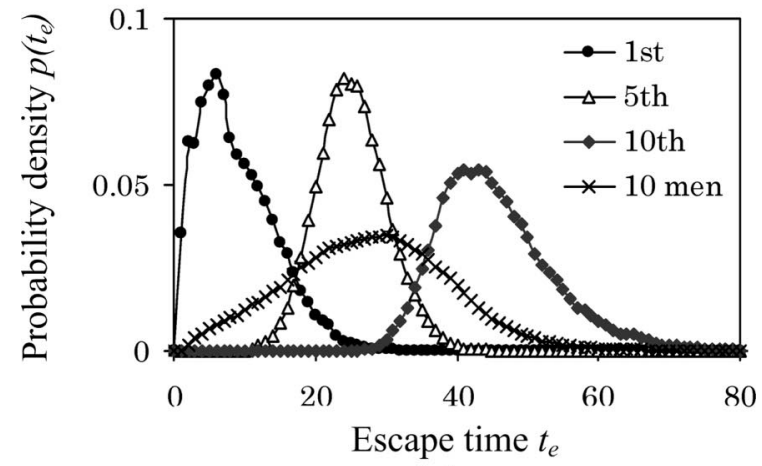

(b)

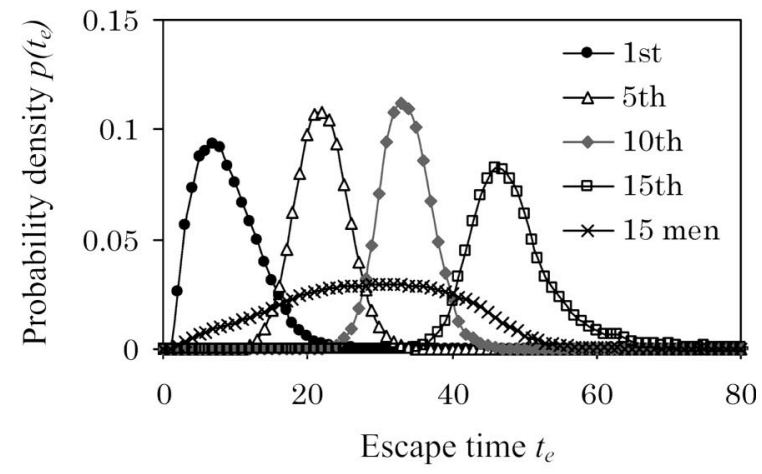

(c)

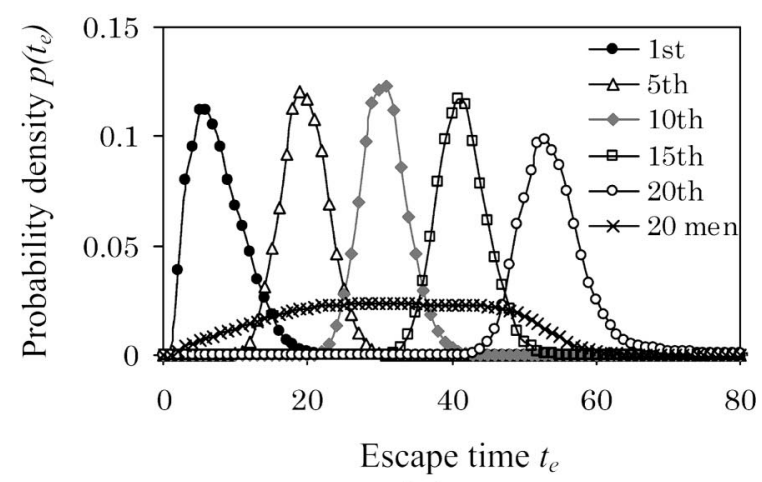

(d)

FIG. 12. Plots of the probability densities of the simulated escape times for (a) 5, (b) 10, (c) 15, and (d) 20 persons. In each figure, the probability density distributions are shown for the 1st, 5th, 10th, 15th, 20th, and all persons.

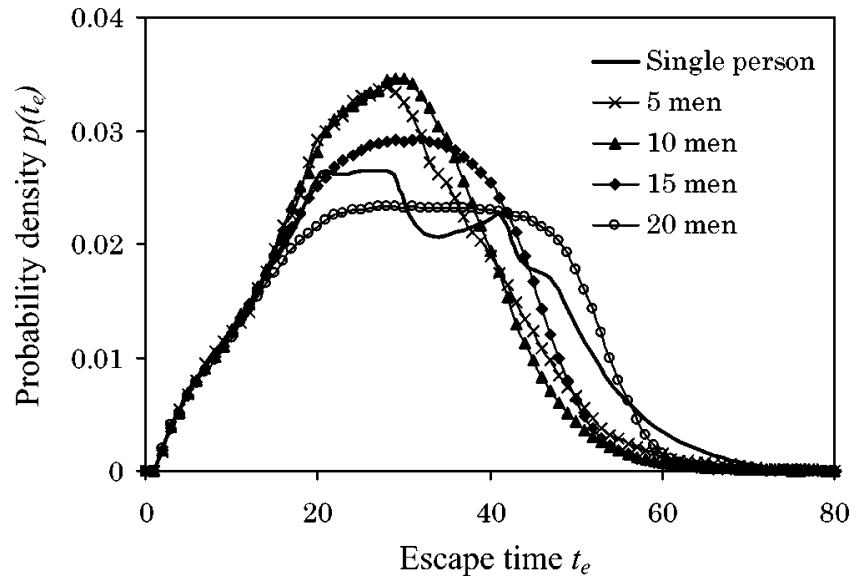

FIG. 13. Probability density distributions of the simulated escape times for all persons in the case of 1, 5, 10, 15, and 20 walkers.

the exit, but the escape is obstructed by the other persons for more than 10 walkers, which is due to jamming near the exit.

Let us now study the simulated escape time of the first walker who successfully escapes from a room with no visibility. Figure 14 shows the plot of the first walker's mean escape time as a function the overall number of walkers initially present in the room. With an increasing number of walkers, the escape time of the first escaped walker decreases. This is because the chances to discover the exit increase with the presence of more people.

\section{SIMULATIONS WITH TWO DOORS}

We will now study the effect of two exits on the evacuation process from a room with no visibility by means of simulations. The second exit is assumed to be located on the opposite side of the room.

The simulations basically agree with the ones for one exit, but we will consider two different cases: In scenario A, as soon as one of the walkers has found an exit, the other walkers are assumed to recognize the location of this exit acoustically and to turn into its direction, as in the scenario with one exit. However, in scenario B we assume that walkers do

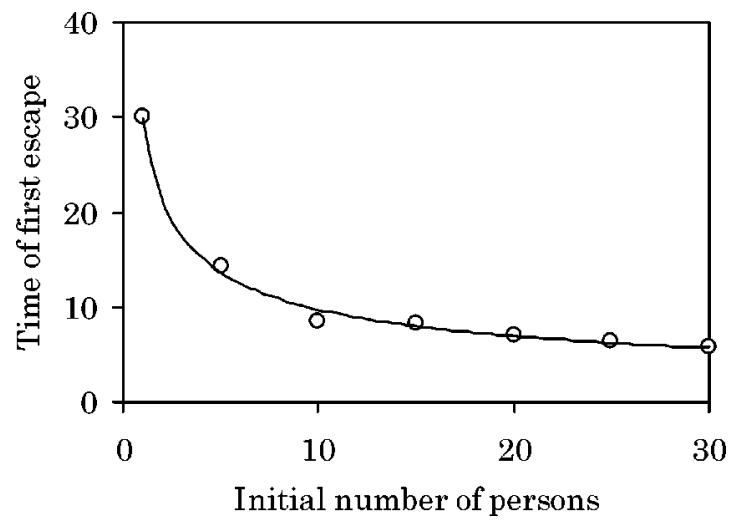

FIG. 14. Plot of the mean escape time of the first escaped walker as a function of the overall number of walkers who are initially present in the room. 


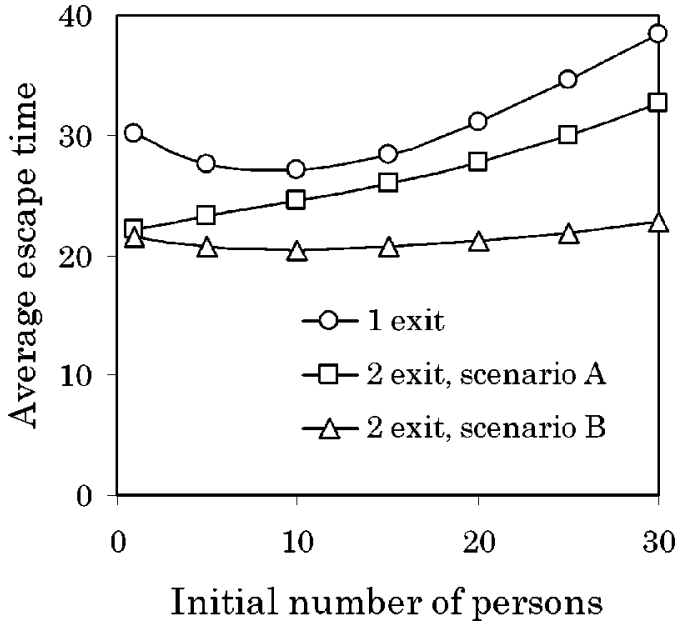

FIG. 15. Plot of the average escape time as a function of the initial number of walkers. Circles represent the results for one single exit. In the two-exit simulation, the average escape time is only reduced a little, as walkers orient towards the exit which has been discovered first, which produces jamming at one door (scenario $\mathrm{A}$, see the squares). The expected significant reduction in the average escape time is only found, if walkers do not react to the discovery of a door by other walkers (scenario B, see the triangles).

not recognize or ignore the discovery of an exit by other walkers.

In scenario A, the existence of two doors reduces the average time until an exit is located first. However, the second door was practically unused, as the walkers turn towards the first discovered exit. One could say that a kind of herding effect is responsible for this. It is established by the nonlocal but direct acoustic interaction when a walker disappears from the room and reinforced by the followers. Consequently, an additional door does not double the flow of escaped persons and does not reduce the average escape time by a factor of 2 , as planners would usually assume. It mainly reduces the exploration time until a door is discovered.

In contrast, in scenario B walkers use the two alternative exits approximately with the frequency that planners usually assume. In some sense, everyone tries to discover the exit by himself. Therefore, the escape time distribution for one person is the same as in scenario A. Nevertheless, people may meet each other and continue their way together. Therefore, we do have interactions in scenario B. Scenario A is more effective in the beginning, when people profit from the information about the location of the exit, while most people in scenario B are still searching. However, as soon as people in scenario A are jamming at one of the exits, scenario B profits from a better usage of all available doors.

Figure 15 shows the average evacuation time for a room with one exit and two opposite exits, respectively. Circles, squares, and triangles indicate the simulation results for one exit, for scenario A, and for scenario B, respectively. Figure 16 displays the overall evacuation time as a function of the initial number of walkers. When the initial number of persons is increased, the difference in the escape times between scenarios A and B becomes large.

In order to decide whether scenario A or B is more realistic, we have performed an experiment for a room with two

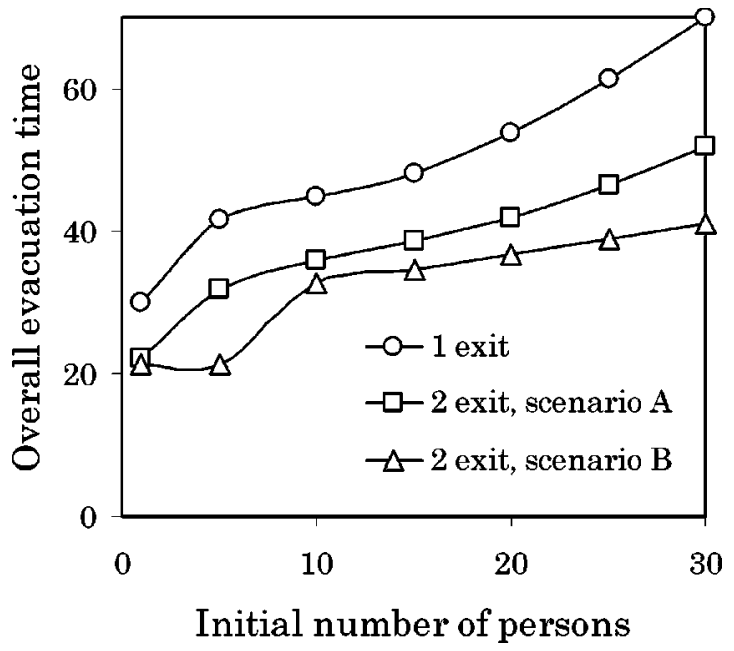

FIG. 16. Plot of the overall evacuation time of all walkers as a function of the initial number of persons. A second exit reduces the overall evacuation time with respect to the situation with one exit (circles), but it does not reduce it by a factor of 2 . If people orient towards the first discovered door (scenario A), the overall escape time is mainly reduced by the earlier discovery of an exit (squares). However, the overall escape time is normally much higher than in the hypothetical scenario B, for which we assume that the discovery of an exit by other people is not recognized or ignored, and both exits are equally used (triangles).

exits for ten persons. We observed that the fractions of persons leaving the two alternative exits were 0.36 and 0.64 . Thus, either the first exit or the second one was used more frequently, which points to a herding effect. However, the difference from 0.5 was not clear enough to exclude scenario B. We assume that the herding effect was less pronounced than expected, because the two doors in our experiment were not located oppositely to each other but on the same side of the room. Therefore, some of the persons turning towards the first discovered exit accidentally passed by the second exit on their way (see also the end of Sec. III for this aspect). Further experiments would be desirable in the future.

\section{SUMMARY AND OUTLOOK}

Focusing on the individual escape times, we have presented experimental results on the evacuation of disoriented students from a classroom with no visibility. The behavior of the disoriented students was very characteristic. They moved slowly towards one of the walls of the room and then along the wall. As soon as one of the persons managed to leave the room, the other ones recognized the location of the exit acoustically and turned into this direction. This reduced the average escape time in the case of one door, as orientation was improved and pedestrians could identify the shorter way to the exit. However, in the case of multiple doors, only the first discovered exit was identified acoustically. People were heading towards this exit and produced unnecessary jamming, as the capacity of one door was not enough to cope with all arriving persons. An acoustic guidance of people by directional sound [29] towards the exits in evacuation situa- 
tions could significantly increase the efficiency of usage of alternative doors, avoid unnecessary jamming, decrease the average escape times, and increase the chances of survival in emergency situations. The advantage of directional sound is that all available exits are recognized and the nearest of several available exits is chosen: i.e., the escaping crowd uses the capacity of all exits. In situations with good orientation, there is generally a tendency to have a load balancing between alternative exits [30].

Despite of the stochastic nature of pedestrian flows, the empirical observations could be semiquantitatively reproduced by an extended lattice gas model-i.e., a stochastic many-particle approach. In particular, we could successfully reproduce the empirically observed behavior of persons in a room without visibility: For example, the average escape time is reduced for ten persons compared to one, as the chances to find the exit increase with the number of persons (see Fig. 14). However, obstructions due to jamming at the exit are expected to dominate for a higher number of persons (see Fig. 13). As a consequence, the model could be used to identify not only the average escape time as a function of the number of people in a dark or smoky room, but it could also help to identify the probability distribution of escape times. This is of practical importance for the assessment of the safety of buildings in emergency situations.
[1] T. Nagatani, Rep. Prog. Phys. 65, 1331 (2002).

[2] D. Helbing, Rev. Mod. Phys. 73, 1067 (2001).

[3] D. Chowdhury, L. Santen, and A. Schadschneider, Phys. Rep. 329, 199 (2000).

[4] B. S. Kerner, Netw. Spatial Econ. 1, 35 (2001).

[5] Traffic and Granular Flow, edited by D. E. Wolf, M. Schreckenberg, and A. Bachem (World Scientific, Singapore, 1996).

[6] D. Helbing and P. Molnar, Phys. Rev. E 51, 4282 (1995).

[7] M. Muramatsu, T. Irie, and T. Nagatani, Physica A 267, 487 (1999).

[8] T. Nagatani, Physica A 300, 558 (2001).

[9] C. Burstedde, K. Klauck, A. Schadschneider, and J. Zittartz, Physica A 295, 507 (2001).

[10] D. Helbing, L. Buzna, and T. Werner, trafficforum/03120401.

[11] J. Dzubiella and H. Löwen, J. Phys.: Condens. Matter 14, 9383 (2002).

[12] J. Dzubiella, G. P. Hoffmann, and H. Löwen, Phys. Rev. E 65, 021402 (2002).

[13] J. Chakrabarti, J. Dzubiella, and H. Löwen, Europhys. Lett. 61, 415 (2003).

[14] Y. Tajima and T. Nagatani, Physica A 292, 545 (2001).

[15] A. Kirchner and A. Schadschneider, Physica A 312, 260 (2002).

[16] H. Klüpfel, M. Meyer-König, J. Wahle, and M. Schreckenberg, in Proceedings of the Fourth International Conference on CA for Research and Industry, edited by S. Babdini and T. Worsch (Springer, London, 2000), p. 63.

[17] D. Helbing, I. Farkas, and T. Vicsek, Nature (London) 407, 487 (2000).

[18] D. Helbing, I. Farkas, and T. Vicsek, Phys. Rev. Lett. 84, 1240 (2000).

[19] M. Muramatsu and T. Nagatani, Physica A 275, 281 (2000).

[20] M. Muramatsu and T. Nagatani, Physica A 286, 377 (2000).

[21] Y. Tajima, K. Takimoto, and T. Nagatani, Physica A 294, 257 (2001).

[22] Y. Tajima and T. Nagatani, Physica A 303, 239 (2002).

[23] T. Itoh and T. Nagatani, Physica A 313, 695 (2002).

[24] S. Maniccam, Physica A 321, 653 (2003).

[25] F. Weifeng, Y. Lizhong, and F. Weicheng, Physica A 321, 633 (2003).

[26] A. Kirchner, H. Klüpfel, K. Nishinari, A. Schadschneider, and M. Schreckenberg, Physica A 324, 689 (2003).

[27] M. Fukui and Y. Ishibashi, J. Phys. Soc. Jpn. 68, 2861, 3738 (1999).

[28] D. Helbing, M. Isobe, T. Nagatani, and K. Takimoto, Phys. Rev. E 67, 067101 (2003).

[29] D. Withington, in Pedestrian and Evacuation Dynamics, edited by M. Schreckenberg and S. D. Sharma (Springer, Berlin, 2002), p. 277.

[30] T. Nagatani et al. (unpublished). 